\title{
Vehicle Axles Disturbing Forces Estimation Based on Ant Colony Optimization Unscented Kalman Filter Algorithm
}

\author{
Wang $\mathrm{Wei}^{{ }^{*}}$, Bei Shaoyi ${ }^{2}$, Zhang Lanchun ${ }^{3}$, Zhu Kai ${ }^{4}$ and Hang Weixing ${ }^{5}$ \\ 1, 2, 3, 4,5 School of Automotive and Traffic Engineering, Jiangsu University of \\ Technology, Jiangsu, Changzhou, 213001, China \\ ${ }^{1}$ nuaawangwei@126.com, ${ }^{2}$ beishaoyi@126.com, \\ ${ }^{3}$ zhanglanchunnuaa@163.com, ${ }^{4}$ zhukaijsut@126.com, \\ ${ }^{5}$ hangweixingjsut@126.com
}

\begin{abstract}
To aim at the problem that lateral disturbance on vehicle are too difficult to measure directly in automatic lane keeping, vehicle axle disturbing forces estimation algorithm was proposed based on vehicle dynamics model and the Ant colony optimization Unscented Kalman filter (AC-UKF) theory. Linear vehicle model of two degree-of-freedom was used, in which lateral forces on front and rear axle were considered. When Unscented Kalman filter (UKF) algorithm was applied to estimate vehicle axle disturbing forces, the Ant colony optimization algorithm was introduced at the same time. The estimation precision increased greatly by using the new adaptive filtering algorithm. Robustness and accuracy of the Ant colony optimization UKF algorithm is verified through the virtual experiment. It can provide theoretic direction for design of estimator in vehicle lateral control system in lane keeping.
\end{abstract}

Keywords: vehicle axles disturbing forces, Ant colony optimization algorithm, UKF algorithm, state estimation, virtual experiment

\section{Introduction}

In the process of driving, the driver invests a lot of time and energy to keep the driveway and it also can cause the driver fatigue. In order to solve this problem, it mainly aims at the study of the auxiliary driving system based on lane keeping. In the early ninety's, the "course control system" was studied based on the experiment by the companies, such as Toyota, BMW, Volkswagen and so on. When the vehicle was divorced from the lane, the system estimated the lateral disturbance online by observer and then steering motion device produced an additional steering torque for vehicle to make it return to the original lane [1]. Thus it can be see that acquiring lateral disturbance accurately and timely is the most basic and important for vehicle automatic lane keeping control, this disturbance is mainly caused by the external environment such as lateral wind or road surface roughness and so on. When a vehicle drives in high speed, the continuous cumulative effects of this disturbance can make the vehicle steering wheel deflect tinyly and out of the reserved lane [2].

In the process of driving, lateral disturbance is difficult to measure directly, therefore estimating the disturbance is the effective way. Current estimation algorithm mainly include linear kalman filter (KF) [3], extended kalman filtering (EKF) [4-5] and Unscented kalman filter (UKF) [6-7], neural network [8-9], the state observer [10] and fuzzy logic [11] and so on. These methods are to estimate and forecast the key control variables of vehicle control system.

${ }^{*}$ Corresponding Author 
This article designs a kind of algorithm called Ant colony optimization unscented kalman filter algorithm, this algorithm will be keeping ant colony algorithm with UKF algorithm and clever union and use ant colony algorithm optimization function to realize algorithmic adaptation and improve the robustness and accuracy of the algorithm. Vehicle axle lateral disturbing force was estimated based on vehicle dynamics model and the Ant colony optimization unscented kalman filter theory. According to the auxiliary measuring variables, minimum variance estimation of the axle disturbance is obtained based on filtering estimation algorithm.

\section{Vehicle Axle Disturbance Estimation Model}

This paper builds vehicle model of two degrees-of-freedom including front and rear axle lateral disturbance force $F_{d f}$ and $F_{d r}$. Here two degree-of-freedom refers to the center of mass of side-slip angle and yawing angular velocity, as shown in Figure1.

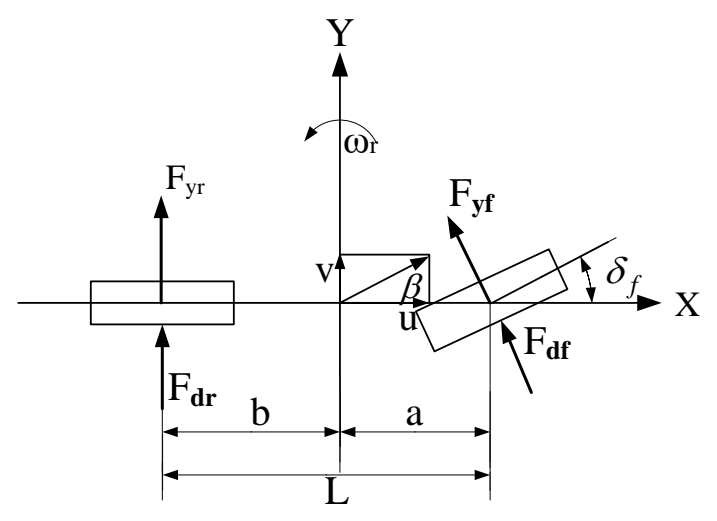

Figure 1. Vehicle Axle Disturbance Model

Because $\delta_{f}$ is small, movement differential equation of vehicle is written as

$$
\begin{gathered}
m\left(\dot{v}+u w_{r}\right)=F_{y f}+F_{d f}+F_{y r}+F_{d r} \\
I_{z} \dot{w}_{r}=\left(F_{y f}+F_{d f}\right) \cdot a-\left(F_{y r}+F_{d r}\right) \cdot b
\end{gathered}
$$

The rate of accelerated change produced by the front axle disturbance $F_{d f}$ is defined as system noise $w_{f}$ and the rate of accelerated change produced by the rear axle disturbance $F_{d r}$ is defined as system noise ${ }^{w_{r}}$, as follow

$$
\begin{gathered}
\dot{a}_{d f}=\frac{\dot{F}_{d f}}{m}=w_{f} \\
\dot{a}_{d r}=\frac{\dot{F}_{d r}}{m}=w_{r}
\end{gathered}
$$

It is assumed that the rate of steering wheel angle change is zero, as $\dot{\delta}=0$.

According to formula (1) and (2), the state equation and measurement equation are written as the following: 


$$
\begin{aligned}
& {\left[\begin{array}{c}
\dot{v} \\
\dot{\omega}_{r} \\
\dot{a}_{d f} \\
\dot{a}_{d r} \\
\dot{\delta}
\end{array}\right]=\left[\begin{array}{ccccc}
a_{11} & a_{12} & 1 & 1 & a_{15} \\
a_{21} & a_{22} & a_{23} & a_{24} & a_{25} \\
0 & 0 & 0 & 0 & 0 \\
0 & 0 & 0 & 0 & 0 \\
0 & 0 & 0 & 0 & 0
\end{array}\right]\left[\begin{array}{c}
v \\
\omega_{r} \\
a_{d f} \\
a_{d r} \\
\delta
\end{array}\right]+G\left[\begin{array}{c}
w_{f} \\
w_{r} \\
0
\end{array}\right]} \\
& {\left[\begin{array}{c}
a_{y} \\
\omega_{r} \\
-\delta
\end{array}\right]=\left[\begin{array}{ccccc}
c_{11} & c_{12} & 1 & 1 & c_{15} \\
0 & 1 & 0 & 0 & 0 \\
0 & 0 & 0 & 0 & -1
\end{array}\right]\left[\begin{array}{c}
v \\
\omega_{r} \\
a_{d f} \\
a_{d r} \\
\delta
\end{array}\right]+\left[\begin{array}{c}
v_{f} \\
v_{r} \\
0
\end{array}\right]} \\
& G=\left[\begin{array}{lll}
0 & 0 & 0 \\
0 & 0 & 0 \\
1 & 0 & 0 \\
0 & 1 & 0 \\
0 & 0 & 1
\end{array}\right], \quad a_{11}=\frac{k_{1}+k_{2}}{m u} \quad, \quad a_{12}=\frac{k_{1} a-k_{2} b}{m u}-u \quad a_{15}=\frac{-k_{1}}{i \cdot m} \\
& a_{21}=\frac{k_{1} a-k_{2} b}{u I_{z}}, \quad a_{22}=\frac{k_{1} a^{2}+k_{2} b^{2}}{u I_{z}} \quad a_{23}=\frac{a m}{I_{z}}, \quad a_{24}=-\frac{b m}{I_{z}} \quad a_{24}=-\frac{k_{1} a}{i I_{z}}, \\
& c_{11}=a_{11}, \quad c_{12}=\frac{k_{1} a-k_{2} b}{m u}, c_{15}=a_{15} \text {. }
\end{aligned}
$$

\section{The Ant Colony Optimization Unscented Kalman Filter Algorithm}

\subsection{The Ant Colony Optimization Algorithm}

The Ant colony algorithm is proposed by an Italian scholars Dorigo based on the route choice behavior of ants foraging. Ants transfer through different sites, transition probability that ants transfer from location $i_{\text {to location }}{ }^{j}$ at time $\mathrm{t}$ can be written as: 


$$
M_{i j}(t)=\left\{\begin{array}{cc}
\frac{\tau_{i j}^{\alpha}(t) \eta_{i j}^{\beta}(t)}{\sum_{r \in \text { Allowed }} \tau_{i r}^{\alpha}(t) \eta_{i r}^{\beta}(t)} & j \in \text { Allowed } \\
0 & \text { Others }
\end{array}\right.
$$

Where $\tau_{i j}(t)$ is the pheromone track strength within the ant's $i$ neighborhood at time $\mathrm{t}, \eta_{i j}$ represents the inspired degree that ants transfer from position $i$ to position $j$, also called visibility, $r$ represents the position that the ants are allowed to reach, Allowed represents the position gather that the ants can choose next, $\alpha$ represents the relative importance of trajectory that the information accumulated by ant colony in the process of movement is the effect on the movements of ant colony. The numerical value is larger, the ants is more inclined to choose other route. $\beta$ represents the relative importance of visibility.

As shown in formula (7), the transition probability is proportional to $\eta_{i j}$ and $\tau_{i j}$. Meanwhile, in the process of optimization, pheromone can be volatile, $\rho \in[0,1)$ is defined as pheromone residual coefficients to show the durability pheromone material and $1-\rho$ is defined as volatility of pheromone. After the completion of a cycle, at time $t+1$ pheromone intensity $\tau_{z \delta}(t+1)$ of Ant colony in the mobile path will be updated and adjusted as the following:

$$
\left\{\begin{array}{c}
\tau_{i j}(t+1)=(1-\rho) \tau_{i j}(t)+\Delta \tau_{i j}(t, t+1) \\
\Delta \tau_{i j}(t, t+1)=\sum_{p=1}^{N} \Delta \tau_{i j}(t, t+1)
\end{array}\right.
$$

Where $\Delta \tau_{i j}(t, t+1)$ is the pheromone that ant $p$ stays on the path in the process of this cycle, the shorter the path, the more pheromone release.

\subsection{The Unscented Kalman Filter Algorithm}

(1) the mean and variance of initialization

$$
\begin{gathered}
\bar{x}_{0}=E\left(x_{0}\right) \\
P_{0}=E\left[\left(x_{0}-\bar{x}\right)\left(x_{0}-\bar{x}\right)^{T}\right]
\end{gathered}
$$

(2) Computing Sigma points $(k=0,1,2 \ldots$. (10).

Creating $2^{n}+1$ Sigma point (columns) composed matrix $\chi_{k}$ based on formula (9) and

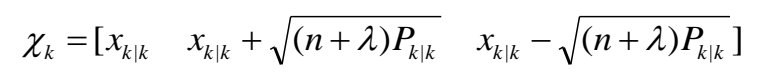

(3) The process of time update

All sigma points are transformed to nonlinear based on nonlinear system state equation:

$$
\chi_{k+1 \mid k}=f\left(\chi_{k \mid k}, u_{k}\right)
$$


State prediction:

$$
x_{k+1 \mid k}=\sum_{i=0}^{2 n} W_{i}^{m} \chi_{i, k+1 \mid k}
$$

Variance matrix prediction:

$$
P_{k+1 \mid k}=\sum_{i=0}^{2 n} W_{i}^{c}\left(\chi_{i, k+1 \mid k}-x_{k+1 \mid k}\right)\left(\chi_{i, k+1 \mid k}-x_{k+1 \mid k}\right)^{T}+Q
$$

All Sigma points are transformed to nonlinear based on observation equation:

$$
\psi_{i, k+1 \mid k}=h\left(\chi_{i, k+1 \mid k}\right)
$$

(4) Observation update

(5) Calculate the system output variance matrix:

$$
P_{y_{k+1} y_{k+1}}=\sum_{i=0}^{2 n} W_{i}^{c}\left(\psi_{i, k+1 \mid k}-y_{k+1 \mid k}\right)\left(\psi_{i, k+1 \mid k}-y_{k+1 \mid k}\right)^{T}+R
$$

Calculate the covariance matrix $P_{x_{k+1} y_{k+1}}$ :

$$
P_{x_{k+1} y_{k+1}}=\sum_{i=0}^{2 n} W_{i}^{c}\left(\chi_{i, k+1 \mid k}-x_{k+1 \mid k}\right)\left(\psi_{i, k+1 \mid k}-y_{k+1 \mid k}\right)^{T}
$$

The filtering gain:

$$
K_{k+1}=P_{x_{k+1} y_{k+1}} P_{y_{k+1} y_{k+1}}^{-1}
$$

Variance update:

$$
P_{k+1 \mid k+1}=P_{k+1 \mid k}-K_{k+1} P_{y_{k+1} y_{k+1}} K_{k+1}^{T}
$$

State update:

$$
x_{k+1 \mid k+1}=x_{k+1 \mid k}+K_{k+1}\left(y_{k+1}-\sum_{i=0}^{2 n} W_{i}^{m} \psi_{i, k+1 \mid k}\right)
$$

\subsection{Determining Ant Colony Algorithm Objective Function Using in the UKF Algorithm Optimization}

Process noise covariance matrix $Q$ is based on UKF algorithm, while the observation noise matrix $R$ is a one dimensional matrix and it is constant. $Q$ and $R$ will be preparative optimization parameters of ant colony algorithm.

After determining the preparative optimization parameters, choosing an appropriate objective function is an important step for ant colony optimization algorithm, it can avoid premature convergence in the process of optimization, so that you can obtain the global optimal solution. The actual variance of information is selected as the objective function, aiming at its minimum value for optimization and it is optimized aiming at its minimum value. 
The objective function is defined as follows,

$$
x_{k+1 \mid k+1}=x_{k+1 \mid k}+K_{k+1}\left(y_{k+1}-\sum_{i=0}^{2 n} W_{i}^{m} \psi_{i, k+1 \mid k}\right)
$$

Where $f$ is the actual variance of information, $\tilde{y}_{k+1 \mid k}$ the information sequence in the process of the UKF filter,

$$
\tilde{y}_{k+1 \mid k}=y_{k+1}-\sum_{i=0}^{2 n} W_{i}^{m} \psi_{i, k+1 \mid k} \text {. }
$$

\section{Algorithms Validation Based on Virtual Test}

In order to verify the feasibility of the proposed method, a certain type of vehicle is simulated on the virtual prototype software ADAMS/Car, vehicle parameters of a certain type of vehicle is used in simulation modeling, the vehicle parameters are written as follows, $m=1685 \mathrm{~kg}, a=1.16 \mathrm{~m}, k_{1}=-6 \times 10^{4} \mathrm{~N} / \mathrm{rad}, i=15$. The vehicle model in ADAMS/Car is divided into suspension, steering system and brake system, body, wheels, the transmission subsystem, etc, corresponding model is established based on the vehicle parameters; Then the input and output signal device "Communicator" is established based on the mutual exchange information between each subsystem and subsystem and test bench provided by ADAMS/Car; Finally the vehicle virtual prototype test model is assembled based on the system, and the model is calibrated and adjusted. Step disturbance $600 \mathrm{~N}$ is stressed at front axle at 0.5 seconds in the process of simulation, rear axle disturbed lags behind the front axle 1.5 seconds, cars make horizontal pendulum angular velocity return to zero via to stress $-3^{\circ}$ angle step input to the steering wheel angle at 4 seconds under the circumstance of disturbance.

The noise lateral acceleration measured values is tested based on virtual test, the lateral acceleration estimated values is obtained based on the UKF filter and the lateral acceleration estimated values is obtained based on the ant colony optimization UKF filter. They are shown in Figure 2. Noisy yawing angular velocity measurement values and yawing angular velocity estimation values based on two algorithm after filtering are shown in Figure 3.

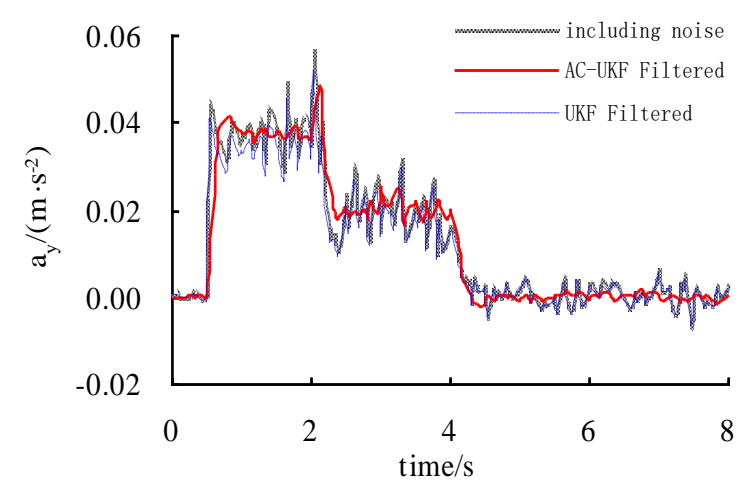

Figure 2. The Lateral Acceleration after Noise and Two Kinds of Algorithm Filtering 


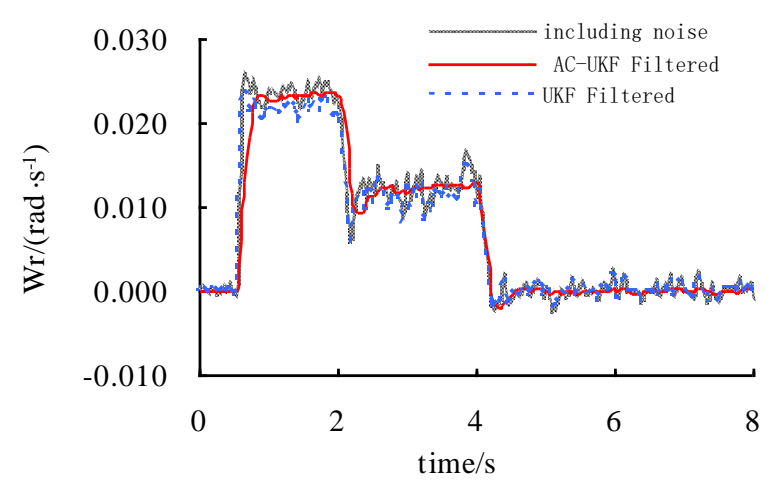

\section{Figure 3. The Yawing Angular Velocity after Noise and Two Kinds of} Algorithm Filtering

We can be seen from Figure 2 and Figure 3, the design of AC-UKF algorithm in this paper relative to use the UKF algorithm for measuring information alone has a very good filtering effect, it can largely eliminate the signal interference caused by the system noise and sensor measurement error.

The actual front axle disturbing force $F_{d f}$ and error variation got based on AC-UKF and UKF estimation are shown in Figure 4 and Figure 5.

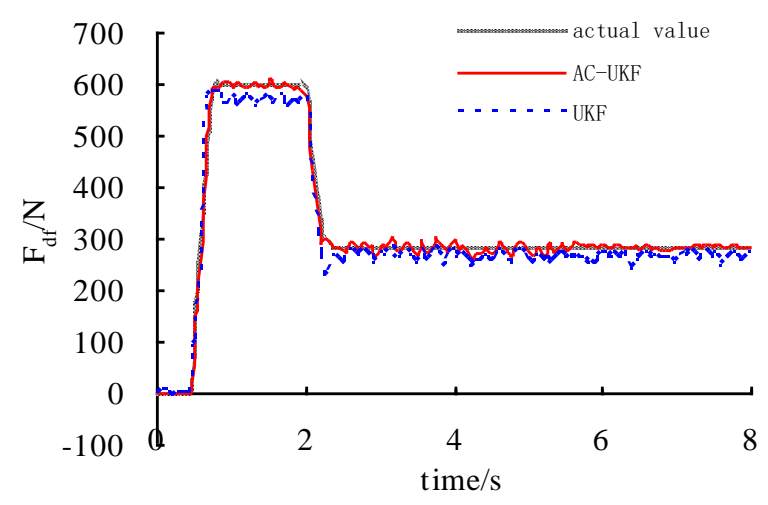

Figure 4. The Actual Front Axle Disturbing Force $F_{d f}$ and Two Kinds of
Algorithm Estimating Value

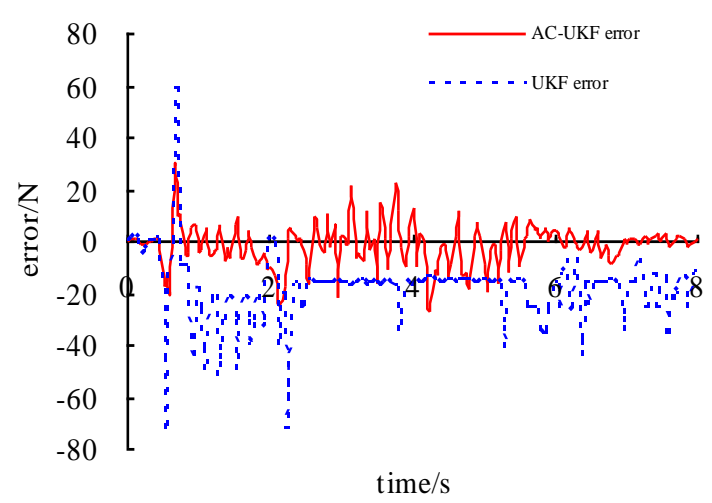

Figure 5. Comparison of Two Algorithms Estimation Error 
Figure 4 and Figure 5 can be seen that the accuracy of front axle interference force estimated by ant colony optimization UKF filter is higher than the accuracy estimated by the UKF filter and it can more keep the trend of step force. The result of AC-UKF estimation has small oscillation after reaching to steady-state. The disturbing force steady-state value estimated comparing to the interference force steady-state value that add in the vehicle front axle in the virtual experiment has a certain error, its value is about $20 \mathrm{~N}$ and this error results from system noise introduced. UKF estimation result has a certain error in the steady-state value and its value is about 50N. Results show that the ant colony optimization UKF estimation precision is higher.

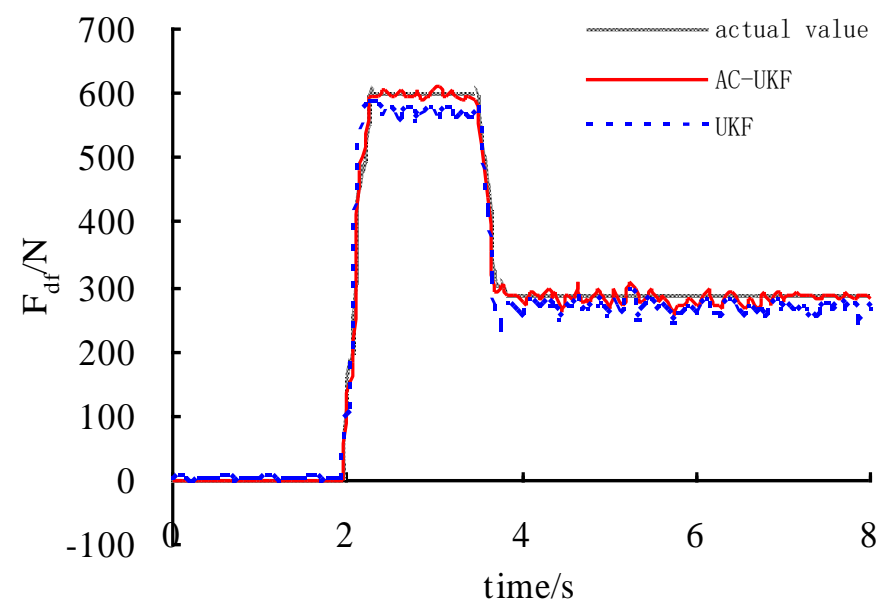

Figure 6. The Actual Front Axle Disturbing Force $F_{d r}$ and Two Kinds of
Algorithm Estimating Value

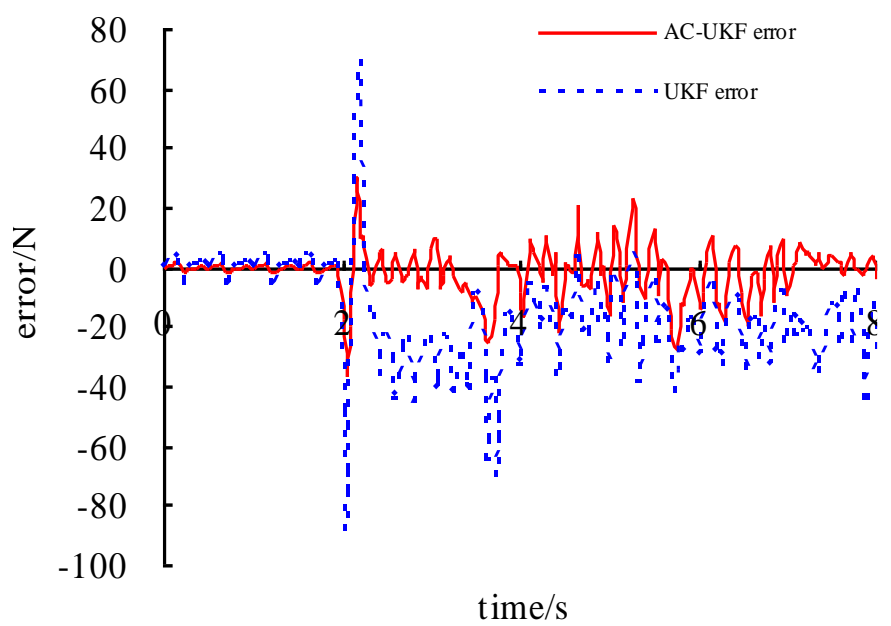

Figure 7. Comparison of Two Algorithms Estimation Error

The step disturbance $F_{d r}$ of rear axle and error variation got based on ant colony optimization UKF and UKF filter estimation are shown in Figure 6 and Figure 7. Figure 6 and Figure 7 can seen that the disturbance tracking capability got based on Ant colony optimization UKF estimation comparing to UKF filter estimate is better and it tallies with the actual disturbance. 


\section{Conclusion}

(1) Vehicle axle disturbing forces estimation is proposed based on the Ant colony algorithm and Unscented Kalman filter theory. UKF algorithm for state estimation will produce large deviation. As the addition of ant colony algorithm, the optimization function gradually reduces evaluated error and it can achieve the effect of high estimation precision.

(2)The virtual test shows that the estimation result of ant colony optimization UKF algorithm can be good to track virtual test values, and the precision of vehicle front and rear axle interference force is higher than that separately estimated by UKF algorithm, it can provide certain theoretical reference for the design of the vehicle lateral control system.

\section{Acknowledgment}

This work was supported in part by the National Science Foundation of China (Grant no. 51305175), the National Science Foundation of JiangSu Province (Grant no. BK2012586) and the National Science Foundation of Jiangsu University of Technology (Grant no. KYY14041).

\section{References}

[1] K. Naab and G. Reichart, "Drive assistance systems for lateral and longitudinal vehicle guidance-heading control and active cruise control", Proceedings of 1994 AVEC. Japan: JSAE, (1994), pp. 449-454.

[2] M. Yamamoto, Y. Kagawa and A. Okuno, "Robust control for automated lane keeping against lateral disturbance", International Conference on Intelligent Transportation Systems. Japan: IEEE, (1999), pp. 240-245.

[3] B. C. Chen and F. C. Hsieh, "Sideslip angle estimation using extended Kalman filter", Vehicle System Dynamics, vol. 46, no. 2008, (2008), pp. 353-364.

[4] L. Xu, S. Xiang and Z. Weigong, "Reliable vehicle state estimation based on improved extended Kalman filter", Journal of Southeast University (Natural Science Edition), vol. 44, no. 4, (2014), pp. 740-744.

[5] Z. Changfu, H. Dan and Y. Xiao, "Vehicle driving state estimation based on extended Kalman filter", Journal of Jilin University (Engineering and Technology Edition), vol. 39, no. 1, (2009), pp. 7-11.

[6] Z. Youqun and L. Fen, "Vehicle State Estimation Based on Unscented Kalman Filter Algorithm", China mechanical engineering, vol. 21, no. 5, (2010), pp. 615-619.

[7] S. Antonov, A. Fehn and A. Kugi, "Unscented Kalman filter for vehicle state estimation", Vehicle System Dynamics, vol. 49, no. 9, (2011), pp. 1497-1520.

[8] Z. Xiaolong, L. Liang and L. Hongzhi, "Experimental Research on Vehicle Sideslip Angle Estimation Based on Improved RBF Neural Networks", Chinese Journal of Mechanical Engineering, vol. 46, no. 22, (2010), pp. 105-110.

[9] S. Melzi and E. Sabbioni, "On the vehicle sideslip angle estimation through neural networks: Numerical and experimental results", Mechanical Systems and Signal Processing, vol. 25, no. 6, (2011), pp. 2005-2019.

[10] F. Cheli, S. Melzi and E. Sabbioni, “An Adaptive Observer for Sideslip Angle Estimation: Comparison With Experimental Results", Proceedings of the ASME 2007 International Design Engineering Technical Conferences \& Computers and Information in Engineering Conference. Nevada: ASME, (2007), pp. 1193-1199.

[11] S. Shuming, L. Henk and B. Paul, "Estimation of Vehicle Side Slip Angle Based on Fuzzy Logic", Automotive Engineering, vol. 27, no. 4, (2005), pp. 426-430. 


\section{Authors}

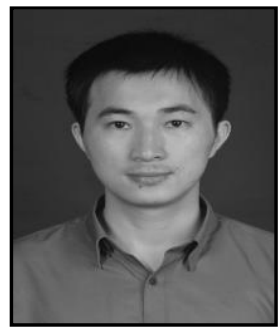

Wang Wei, he is a lecture at School of Automobile and Traffic Engineering, JiangSu University of Technology (JSUT). He was born in 1984. He received doctor degree in Vehicle engineering from Nanjing University of Aeronautics and Astronautics(NUAA), China in 2014. He is engaged in the scientific research and the teaching work.

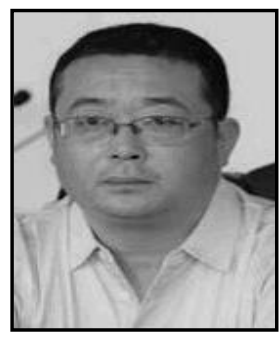

Bei Shaoyi, he is a professor at School of Automobile and Traffic Engineering, JiangSu University of Technology (JSUT). He was born in 1968. He received doctor degree in Vehicle engineering from Jiangsu University.

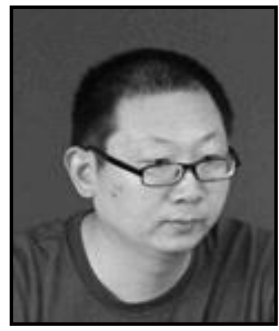

Zhang Lanchun, he is an associate professor at School of Automobile and Traffic Engineering, Jiangsu University of Technology (JSUT). He was born in 1980.He received doctor degree in Vehicle engineering from Nanjing University of Science and Technology(NUST), China in 2009.

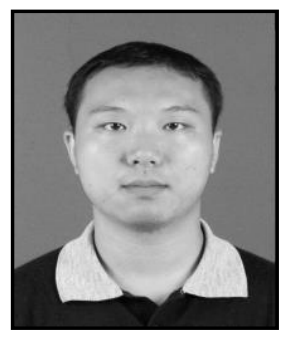

Zhu Kai, he is a lecture at School of Automobile and Traffic Engineering, Jiangsu University of Technology (JSUT). He was born in 1984.He received doctor degree in Control engineering from Nanjing University of Science and Technology(NUST), China in 2015.

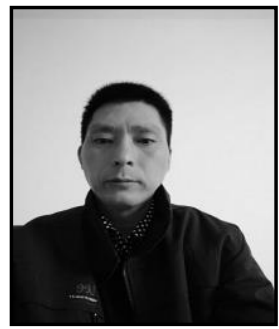

Hang Weixing, he is a lecture at School of Automobile and Traffic Engineering, University of Technology (JSUT). He was born in 1972.He received bachelor degree in Vehicle engineering from Yangzhou University. 